\title{
Cognition in Pediatric SDB - Yes, No, Maybe?
}

\author{
Scott Hunter $^{1}$ and Ariana Garagozzo ${ }^{2}$ \\ ${ }^{1}$ The University of Chicago \\ ${ }^{2}$ Roosevelt University
}

February 15, 2021

\begin{abstract}
Numerous studies in the past 10 years have reported on the neurocognitive sequalae of pediatric Sleep Disordered Breathing (SDB). Variations in criteria used to define SDB in conjunction with the wide variety of neuropsychological measures selected to evaluate cognitive consequences of SDB have resulted in discrepancies within the literature. This review summarizes the extant literature regarding cognitive effects of pediatric SDB across domains of global intelligence, attention, executive function, memory, language, and visuospatial ability. This review also addresses the proposed etiology underlying neurocognitive consequences of pediatric SDB. The differences in findings across the literature are highlighted and discussed throughout.
\end{abstract}

\section{Introduction}

Sleep disordered breathing (SDB) represents a wide spectrum of respiratory abnormalities in sleep. These abnormalities range in severity from Primary Snoring (PS) to Obstructive Sleep Apnea (OSA). PS is characterized by vibrations of tissue in the upper airway that occur during sleep without accompanying apnea or hypopnea. ${ }^{1}$ Prevalence rates of PS are estimated at $6 \%$ of school aged children. ${ }^{2}$ OSA is characterized by narrowing or collapse of the airway during sleep, which results in episodic airway obstruction, hypoxia, and subsequent arousal. ${ }^{3}$ Repeated arousals are associated with disrupted sleep architecture, which can result in non-restorative sleep. OSA occurs in approximately $3 \%$ of school aged children. ${ }^{4}$ Currently, the gold standard assessment for the diagnosis of OSA is polysomnography (PSG), which identifies disruptions in sleep stages and cycles. ${ }^{1}$

There are multiple risk factors associated with pediatric SDB. Commonly cited risk factors in the United States include male gender ${ }^{5}$ and African American race. ${ }^{5}$ One study reported that African American children were three and a half times more likely to have SDB than white American children. ${ }^{6}$ Children born before 34 weeks of gestational age and children with asthma ${ }^{6-8}$ are also at a heightened risk for SDB. ${ }^{9-10}$ Obesity, as indicated by body mass index (BMI $)^{11-13}$ or adipose tissue deposits ${ }^{5}$ is an additional significant risk factor.

There is an extensive body of literature examining the neurocognitive sequalae of SDB in children. This review focuses on literature regarding the cognitive effects of pediatric SDB and the proposed etiology underlying neurocognitive consequences of pediatric SDB. Current discrepancies and limitations of the literature are discussed.

\section{Global Intelligence}

The majority of studies investigating global intelligence of children with SDB report full-scale IQ scores ${ }^{9,}$ 14-15 or general conceptual ability scores ${ }^{11,13,16-17}$ in the average range compared to normative data. These findings are largely consistent among preschool, ${ }^{15,18-19}$ school-aged, ${ }^{14,16,20}$ and adolescent ${ }^{21}$ children across the Wechsler Abbreviated Scale of Intelligence, ${ }^{14}, 20,22$ Wechsler Intelligence Scale for Children, Third Edition (WISC-III) ${ }^{16,23}$ and Fourth Edition (WISC-IV) ${ }^{21,}{ }^{24}$, the Stanford Binet, ${ }^{18,}{ }^{25}$ Kaufman Assessment Battery for Children, ${ }^{9,26}$ and Wechsler Preschool and Primary Scale of Intelligence, Third Edition. ${ }^{15,} 27$ While 
multiple studies controlled for race ${ }^{11,21,28-29}$ and socioeconomic status, ${ }^{15,21,29}$ only one study highlighted demographic-based differences; Hunter et $\mathrm{al}^{11}$ reported that African American children had more symptoms of SDB than White American children.

Studies that implemented control groups reported that global intelligence scores are reduced in children with SDB compared to controls. ${ }^{5,}$, $, 14,17,19$ In some studies, this effect was driven by differences in performance on non-verbal intelligence tasks ${ }^{17}, 19$ while in other studies the effect was driven by differences in verbal intelligence tasks ${ }^{14}$ or unspecified differences in global cognitive function. ${ }^{21,}{ }^{28}$ Therefore, while children with SDB score in the broadly average range on neuropsychological measures of intelligence, they perform lower than children in control groups. This discrepancy is partially explained by the definition of average according to the normal curve; normative data indicates that average abilities range from the $25^{\text {th }}$ to the $75^{\text {th }}$ percentile. Therefore, while children with SDB and children in control groups both demonstrate average intelligence according to normative data, children with SDB score on the lower end of average compared to controls.

To further explore the cognitive profiles of children with SDB, differences in global intelligence across SDB severity groups have been investigated. One study compared children with moderate SDB (AHI [?] 5 per hour) to children with mild SDB (AHI [?] 4 per hour) and reported lower global intelligence scores in the moderate SDB group. ${ }^{16}$ Other studies compared global intellect between children with OSA and children with PS and reported greater deficits in children with OSA. ${ }^{11,28}$ However, findings regarding significance of SDB severity level are not consistent across all studies. A large group of studies reported no differences in global intelligence across SDB severity groups. ${ }^{14,15,18,21,29}$ Three out of the five studies that reported no differences were conducted with infants and pre-school aged children. ${ }^{15,}{ }^{29}$ It is possible that short-term sequelae of SDB in children this age are not severe enough to elicit impairment on objective neuropsychological measures. Therefore, differences in neurocognitive abilities may be more readily observed with prolonged exposure to intermittent hypoxia. ${ }^{15}$

To measure the impact of SDB on global intellect, multiple studies investigated associations between respiratory factors and standardized intelligence scores. Some studies that investigated associations between PSG parameters such as arousal index, obstructive apnea hypopnea index and oxygen saturation reported no associations with global intellect, ${ }^{5,14,15,18}$ indicating that changes in respiratory status were not related to cognitive impairment. Other studies that measured similar PSG parameters reported that total arousal index, ${ }^{17}$ apnea hypopnea index (AHI), ${ }^{11}$ and snoring status ${ }^{30}$ correlated negatively with global intelligence scores. These results suggest that higher rates of respiratory distress are related to decreased intellectual functioning in children with SDB.

In summary, research on the global intellectual profile of children with SDB is discrepant. Some of the studies reviewed above were limited by small sample sizes and a lack of a control group. ${ }^{16,}{ }^{20}$ Almost all studies defined SDB severity using different criteria; some studies defined SDB severity in terms of mild, moderate, or severe OSA, ${ }^{14,15,18}$ while others defined SDB severity according to the presence of snoring with or without associated apneic events. ${ }^{5}$ Additional discrepancies include lack of uniformity for measures of intellectual functioning, which complicated the amalgamation of findings. In regard to referral sources, some of the studies reviewed above included participants who were referred for concerns regarding SDB, ${ }^{14}, 15,16,18,20$ while others included participants recruited from the general population. ${ }^{5,11,17,29}$ As noted by Smith et al ${ }^{30}$ participants referred from medical clinics likely differ from those recruited within the community. Finally, some studies did not control for covariates such as socioeconomic status (SES) ${ }^{11,20}$ or BMI, ${ }^{16,20}$ which are known correlates of SDB. These discrepancies complicate the interpretation of the impact of SDB on global cognitive functioning.

\section{Language}

Discrepant findings have been reported in studies investigating the impact of pediatric SDB on language. In comparison to normative data, children with SDB have average expressive language abilities. ${ }^{11,16-17}$ These findings are consistent across multiple language measures, including but not limited to the WISC-III, ${ }^{16,23}$ Expressive Vocabulary Test, ${ }^{11,31}$ and NEPSY: A Developmental Neuropsychological Assessment ${ }^{32}$ Language 
Domain. ${ }^{17}$ Findings regarding receptive language in this population are mixed. The majority of studies reported average receptive language abilities across multiple measures, including but not limited to the Peabody Picture Vocabulary Test ${ }^{11,33}$ and NEPSY ${ }^{32}$ receptive language tasks. ${ }^{11,18,34-35}$

Despite broadly average performance on language tasks in children with SDB compared to normative data, differences emerge when comparing language abilities in children with SDB to control groups. Multiple studies reported greater language impairment in children with SDB on expressive ${ }^{29}$ and receptive $e^{9,17-18,29-30,34}$ language tasks compared to controls. One study that followed infants with SDB over time reported that at two years of age, children with persistent SDB symptoms scored 5.3 points lower on a measure of language compared to children without SDB symptoms. ${ }^{29}$ Other studies reported no differences on language performance across pediatric SDB and control groups. ${ }^{18,34}$

Limited research has investigated differences in expressive and receptive language performance across multiple SDB severity levels in children. Hunter et $\mathrm{al}^{11}$ reported that children with moderate to severe SDB (AHI $>5$ ) performed worse on a NEPSY receptive language task than children with less severe SDB. The same study reported no differences across SDB severity levels on other language tasks. ${ }^{11}$ Biggs et al ${ }^{18}$ similarly reported no significant differences across SDB severity level and language performance.

To determine the impact of SDB on language, research has explored the associations between respiratory status and language performance. Results indicate that a number of respiratory indicators, including the respiratory disturbance index, ${ }^{18}$ oxygen desaturation, ${ }^{36}$ sleep duration,${ }^{29}$ and frequency of snoring ${ }^{9}$ were associated with poorer expressive ${ }^{29,36}$ and receptive $e^{9,18,29}$ language performance in children with SDB.

The studies reviewed above are limited by their examination of language as a broad construct. Future studies should expand on language profiles in children with SDB by incorporating measures of articulation, auditory processing, and social pragmatics. In addition, the literature reviewed above is characterized by discrepancies regarding language development in children with SDB. Definitions of SDB and SDB severity varied across studies. For example, one study measured SDB with a composite score that combined respiratory variables, ${ }^{17}$ while another measured SDB according to AHI. ${ }^{18}$ Without consistent SDB indicators and severity categories, it is challenging to meaningfully compare results across studies. Additionally, not all studies measured important covariates such as SES ${ }^{11,36}$ and BMI, ${ }^{16,36}$ which are known to influence SDB and cognitive performance.

\section{Memory}

Discrepant findings were observed across studies that investigated memory performance in children with SDB. These results were observed across a range of memory measures, such as the Children's Memory Scale, ${ }^{20-21,37}$ California Verbal Learning Test, ${ }^{21,38}$ and NEPSY ${ }^{32}$ memory tasks. ${ }^{17,19,39-40}$ Children with SDB performed in the average range on working memory tasks. ${ }^{17,20}$ On verbal memory tasks, multiple studies indicated average encoding, ${ }^{17,21}$ consolidation, ${ }^{17,21}$ short-term retrieval, ${ }^{17,21}$ long-term retrieval, ${ }^{21}$ and recognition abilities ${ }^{17,21}$ across list learning ${ }^{21}$ and story learning tasks. ${ }^{17,21}$ Multiple studies suggested similarly average encoding, consolidation, short-term retrieval, and long-term retrieval abilities on visual memory tasks. ${ }^{17,20}$ Despite broadly average performance, some studies that included a control group reported differences in memory abilities for children with and without SDB. ${ }^{19,39-40}$ These differences were observed across verbal memory tasks, ${ }^{19}$ visual memory tasks, ${ }^{19}$ and tasks with both verbal and visual components. ${ }^{39-40}$ Kheirandish-Gozal et $\mathrm{al}^{39}$ measured non-verbal memory with four learning trials, an immediate recall trial, and a delayed recall trial and reported that children with SDB had lower overall performance on the first trial and over all four total trials compared to children without SDB. In addition, children with SDB had lower performance on the immediate and delayed recall trials compared to children without SDB. ${ }^{39}$ These results indicate that children with SDB may have difficulties with the acquisition and retention of new information. A more recent study supported these findings by reporting that children with OSA had poorer overall memory consolidation than controls on a spatial declarative memory task. ${ }^{40}$

Limited research has investigated differences of SDB severity level on memory performance. One study of SDB in school-aged children reported slight differences according to SDB severity; children who had mild 
SDB performed higher than children with moderate SDB on a narrative memory measure. ${ }^{16}$ Another study of SDB in overweight adolescents reported no differences among individuals with primary snoring, mild, or moderate SDB on verbal memory. ${ }^{21}$

To determine the impact of SDB on memory, multiple studies investigated associations between respiratory indices and memory performance. Gregory et $\mathrm{al}^{41}$ conducted an eight-year longitudinal study and reported that sleep problems at ages five, seven, and nine were not associated verbal memory performance at age 13. Kheirandish-Gozal et $\mathrm{al}^{39}$ reported that the respiratory arousal index predicted the slope of learning and delayed recall in children with SDB. These results indicated that sleep fragmentation may influence the challenges that children with SDB have when selecting efficient learning strategies. ${ }^{39}$ A more recent study identified specific sleep architecture features associated with NREM stage two sleep in SDB that might influence memory consolidation. ${ }^{40}$ In healthy children, sleep-dependent memory consolidation is associated with NREM stage two (N2) sleep spindles. N2 sigma power is positively associated with memory consolidation. ${ }^{40}$ In this study, children with OSA had lower N2 sigma power than children with PS and controls. ${ }^{40}$ Therefore, N2 sigma power might serve as a biomarker of neurocognitive challenges in pediatric SDB.

The literature reviewed above revealed multiple discrepancies regarding memory performance in children with SDB. A number of the studies were limited by small sample sizes. ${ }^{40}$ In addition, most of these studies defined SDB and SDB severity with different criteria, making it challenging to compare results across studies. While some of these studies recruited participants from the general population, ${ }^{17,19}$ others included clinical samples of children referred for concerns regarding SBD, ${ }^{16,20,39-40}$ which may create potential referral bias.

\section{Visuospatial Ability}

In comparison to normative data, multiple studies reported average visuospatial skills in children with SDB. ${ }^{16-18}$ Reports of average performance remained consistent across multiple tests, including the Beery Visual Motor Integration System, ${ }^{16,42}$ the NEPSY ${ }^{32}$ Visuospatial domain, ${ }^{17-18}$ and the McCarthy Perceptual Performance task. ${ }^{16,43}$ Few studies utilized a control group or investigated differences in visuospatial abilities across SDB severity levels. Biggs et $a^{18}$ investigated differences in visuospatial ability across SDB severity levels and reported no differences. While the majority of studies on SDB and visual perception are crosssectional, one study investigated visuospatial skills over time and reported no differences in abilities between children with resolved or unresolved SDB after three years. ${ }^{18}$

Studies that investigated associations between respiratory indices and cognition in children with SDB reported no relationship between oxygen desaturation or acute respiratory index and visuospatial abilities. ${ }^{18}$ One longitudinal study indicated that parent report of persistent sleep problems at age five years and age nine years predicted poorer scores on visuospatial tasks at age thirteen years. ${ }^{41}$ Therefore, it may be beneficial to include variables of sleep duration when investigating associations between SDB and visuospatial abilities. Understanding the impact of SDB on visuospatial skills is limited by a focus on general visuospatial constructs, rather than more detailed components of visual discrimination, form constancy, visual figure-ground, and visual closure. Additional limitations include small sample sizes ${ }^{36}$ discrepant criteria for defining SDB and SDB severity levels, and inconsistency in measures of visual perceptual abilities across all studies.

\section{Attention}

Attention is broken down into components of selective attention, which reflects our ability to focus on particular events, divided attention, which refers to our ability to attend to two stimuli simultaneously, and sustained attention, which refers to our ability to focus on activities for a long period of time. ${ }^{44}$ In comparison to normative data, studies reported average simple attention ${ }^{16,41}$ and below average sustained attention ${ }^{9,16,20}$ for children with SDB. Studies that measured sustained attention with continuous performance tasks reported borderline impaired overall performance ${ }^{19}$ and $30 \%$ more errors of commission. ${ }^{20}$ Studies that differentiated between auditory and visual attention reported below average scores in auditory attention ${ }^{20}$ and low average $^{20}$ to average ${ }^{11,17}$ scores in visual attention. Limited research has investigated SDB severity level on simple and sustained attention. Hunter et $\mathrm{al}^{11}$ reported no attentional differences among snoring children, 
children with mild SDB, or children with moderate to severe SDB. More research is required to explore the effects of SDB severity on attentional capacities, both globally and more specifically.

There has been limited research on the association between respiratory characteristics and attention. Chervin ${ }^{45}$ reported no associations between attention problems and SDB status or PSG assessment characteristics. In contrast, Christiansz et $\mathrm{al}^{46}$ reported significant associations between attention and respiratory parameters, such as slow wave activity (SWA), which is a sensitive indicator of sleep disruption. Higher levels of SWA were associated with an increase in percentage of sustained attention errors. ${ }^{46}$

Discrepancies in the research on attention and SDB are likely influenced by small sample sizes, ${ }^{16,20}$ lack of control groups, ${ }^{16,20}$ differences in definitions of SDB and SDB severity level, and variations between community based $^{11,17,19}$ and clinic based ${ }^{6,20,46}$ referrals. In addition, different measures of attention were implemented across the majority of studies, making it difficult to effectively compare results.

\section{Executive Function}

Executive function (EF) is a broad domain that includes skills to guide goal-directed behavior, including the ability to shift tasks, update information into working memory, and inhibit responses. ${ }^{47-48} \mathrm{EF}$ has been well-researched in children with SDB. ${ }^{11,14,16-17,20,21,46}$ Compared to normative data, most studies report average abilities to plan and monitor tasks, ${ }^{11,17,21}$ think flexibly, ${ }^{16}$ problem-solve effectively, ${ }^{20}$ and sequence tasks. ${ }^{21}$ Other studies reported impairments in these same domains. ${ }^{14,46}$ One recent study reported that children with SDB fell in the bottom $25 \%$ on tasks that measured mental flexibility and problem solving compared to normative data. ${ }^{46}$ Another study supported these results with findings of reduced problemsolving abilities in children with SDB, compared to normative data. ${ }^{14}$

Multiple studies that included a control group reported differences in EF capabilities for children with and without SDB. In some studies, SDB groups performed worse than controls in general attention/EF domains, ${ }^{17,19}$ specific planning and monitoring tasks, $,{ }^{9}, 17$ and efficiency tasks. ${ }^{14}$ Other studies found no differences on measures of planning, ${ }^{14,21}$ organization, ${ }^{14,21}$ and verbal fluency. ${ }^{14} \mathrm{~A}$ recent meta-analysis investigated differences in EF between children with SDB and controls. They reported a medium effect size in the domain of generativity. ${ }^{49}$ No significant differences were found on standardized measures of inhibition, working memory, shifting, or vigilance. ${ }^{49}$

Studies that investigated the influence of SDB severity level on EF reported more errors on cancellation tasks in children with mild, compared to moderate SDB, ${ }^{16}$ and greater impairment on problem-solving tasks in individuals with severe compared to moderate or mild SDB. ${ }^{11}$ Others reported no differences across SDB severity levels for sequencing, ${ }^{21}$ planning, ${ }^{11,14}$ and organizing. ${ }^{11,14}$ Therefore, preliminary research suggests that children with all levels of EF severity may demonstrate reduced executive abilities in domains including but not limited to planning, organizing, inhibition, and problem solving.

Numerous studies investigated associations between SDB respiratory parameters and EF deficits. Some studies reported no association between respiratory parameters and EF. ${ }^{5,14}$ Other studies reported that a higher arousal index was associated with decreased mental flexibility, ${ }^{20}$ planning, ${ }^{17}$ organization, ${ }^{17}$ difficulty solving complex tasks,${ }^{46}$ and increased reaction time. ${ }^{46}$ Additional respiratory parameters such as AHI, ${ }^{20}$ slow wave activity, ${ }^{46}$ and oxygen desaturation ${ }^{46}$ have also been related to executive dysfunction in this population. Taken together, the literature indicates that sleep duration and indices of respiratory status are associated with executive dysfunction across multiple domains in children with SDB.

Kaihua et $\mathrm{al}^{50}$ investigated indicators of executive dysfunction in children with SDB using event related potentials (ERP). Using an electroencephalograph (EEG), the authors measured two constituents of ERP (N2 and P3 amplitude and latency) while children were completing a Go/No Go task. ${ }^{50}$ During the Go task, children with SDB demonstrated elevated amplitude and latency of P3, indicating a higher level of attentional investment and delayed response time compared to controls. ${ }^{50}$ During the NoGo task, children with SDB demonstrated reduced N2 amplitude and increased P3 latency, indicating reduced impulse inhibition and longer response time compared to controls. ${ }^{50}$ This suggests that children with SDB require additional time 
and additional attentional capacity to complete inhibitory tasks, compared to children without SDB. ${ }^{50}$ The authors conjectured that intermittent sleep and hypoxia might influence neural circuitry in children with SDB, contributing to the increasing amplitude and latency of P3 and decreased amplitude of N2. ${ }^{50}$

There were large discrepancies in the literature regarding the impact of SDB on EF. These discrepancies were likely influenced by limited sample sizes, ${ }^{16,20,50}$ a large variety of EF measures, wide ranging discrepancies in SDB definitions and criteria, and lack of control for important covariates including BMI ${ }^{16}$ and SES. ${ }^{11}$ In addition, multiple studies recruited participants at-risk for SDB through clinical referrals ${ }^{14,16,20,46,50}$ rather than the community. ${ }^{11,17,19}$

\section{Interplay of Cognition, Emotion, and Behavior}

It is important to note that cognitive challenges occur in the context of other contextual and health variables. ${ }^{51}$ In addition to effects of SDB on cognition, there is a large body of research related to the effects of SDB on behavior, which is beyond the scope of this article. For a systematic review of SDB and behavior the reader is directed to da Silva Gusmão Cardoso, Pompèia, and Miranda. ${ }^{52}$ However, because these intersections are an important consideration, we address the relationship as pertinent to our discussion on cognition. Previously, researchers suggested that behavioral challenges might mediate the relationship between SDB and cognitive functioning. ${ }^{21}$ Smith and colleagues ${ }^{53}$ investigated the indirect effects of SDB on cognition through behavior and reported that behavioral problems mediated the relationship between SDB status and cognition. Therefore, SDB status was related to greater behavioral problems, and greater behavioral problems were related to cognitive functioning. ${ }^{53}$ One notable limitation of this study was that it did not control for SES or prematurity status, which are often associated with SDB and cognitive performance. ${ }^{53}$

In addition to the interplay between cognition and behavior, studies have researched relationships among SDB and emotion. While the broader relationship between SDB and emotional problems in children is not the focus of this paper, and the reader is directed to Liu, et $\mathrm{al}^{54}$ for a comprehensive review regarding this topic, we briefly describe the complex intersections of cognition, emotion, and SDB. Stojek and colleagues ${ }^{55}$ investigated the relationship among fitness, depression, and attention in children with SDB and found that SDB directly mediated the relationship between fitness and depression, such that less fitness was related to greater SDB, which was in turn related to higher levels of depression. ${ }^{55}$ In addition, the authors reported that SDB mediated the relationship between fitness and attention, meaning less fitness was associated with greater SDB, which was associated with worse performance on attentional measures. ${ }^{55}$ Additional research is needed to support ideas regarding the intersection of SDB, cognition, emotion, and behavior.

\section{Underlying Etiology}

There are several hypotheses regarding the mechanisms that underlie cognitive difficulties in children with SDB. Studies have suggested that cognitive sequalae are a product of episodic decreases in oxyhemoglobin saturation, ${ }^{11,17}$ disrupted sleep continuity, ${ }^{11,17}$ and disrupted sleep architecture ${ }^{17}$ that occur in children with SDB. These changes are thought to affect children across the spectrum of SDB severity, including children with PS. ${ }^{11}$ Some studies suggest that the stress of intermittent hypoxia and sleep disruption may elicit inflammatory markers. ${ }^{5,28}$ A high-sensitivity C-reactive protein (hs-CRP), which measures general levels of inflammation in the body, is elevated in children with OSA compared to children without OSA. ${ }^{28}$ Hs-CRP is also elevated in children with OSA who have cognitive deficits compared to children with OSA who do not have cognitive deficits. ${ }^{28}$ Therefore, systemic inflammation may increase neurocognitive impairment in children with SDB. An additional study that investigated the role of increased adiposity on cognitive abilities in children with SDB reported that increased central adiposity influences cognition through mechanisms of low grade, chronic inflammation. ${ }^{5}$ Therefore, obesity and SDB can both contribute to chronic inflammatory responses, which are associated with decreased cognitive abilities in some children with SDB. ${ }^{5,28}$

Since increased inflammation is not associated with decreased cognition in all children with SDB, there is likely a combination of genetic and environmental factors that interact to elicit neurocognitive risk. Polymorphisms in the NOX gene or its subunits ${ }^{56}$ as well as apoliprotein E allelic variants ${ }^{57}$ have been implicated in underlying gene-related susceptibility for neurocognitive deficits in children with SDB. Therefore, some 
children may have a unique and complex SDB phenotype that predisposes them to cognitive challenges.

\section{Conclusions}

Children with SDB demonstrate broadly average cognitive abilities in comparison to normative data across global intellectual functioning, memory, attention, EF, and language domains. ${ }^{9}{ }^{14-16,18-21,29}$ However, these children often perform significantly worse on measures of cognitive functioning when compared to controls without SDB..$^{9,14,17,19,28}$ These differences were often observed across the SDB spectrum, including children with PS or mild OSA, indicating that low levels of SDB increase risk for cognitive difficulties. ${ }^{11,16,28,30}$

It is critical to consider contextual variables when interpreting the effects of SDB on cognition. Factors such as chronic health conditions and perceived discrimination, which indirectly influence cognition for African Americans ${ }^{51}$, were not included in any of the reviewed studies. Future research should include known predictors of health disparities when investigating the relationship of SDB on cognition. Future research should also explore the above findings across additional cultural contexts.

The underlying mechanisms for cognitive consequences of SDB are still being investigated. Research supports the role of disrupted sleep architecture and intermittent hypoxia on chronic inflammation, which is associated with cognitive deficits. ${ }^{5,28}$ There appears to be an interaction between genetic susceptibility and environmental factors, which may explain why some children with SDB have cognitive impairments, while others do not. ${ }^{56-57}$ Further research is needed to strengthen our understanding of the mechanisms that underlie the neurocognitive profiles of children with SDB.

Clinicians, educators, and individuals from the general population must be educated on SDB and the neurocognitive sequalae of SDB in order to increase rates of early identification and early care.

\section{Conflicts of Interests}

The authors declare that there are no conflicts of interests.

\section{Author Contributions}

Ariana Garagozzo conducted the original literature search and wrote the original draft preparation. Scott Hunter conducted an additional literature search, reviewed, and edited iterations of the manuscript.

References

1. Marcus CL, Brooks LJ, Draper KA, et al. Diagnosis and management of childhood obstructive sleep apnea syndrome. Pediatrics . 2012;130(3):e714-755. doi:10.1542/peds.2012-1672

2. Brockmann PE, Urschitz MS, Schlaud M, Poets CF. Primary snoring in school children: Prevalence and neurocognitive impairments. Sleep Breath . 2012;16(1):23-29. doi:10.1007/s11325-011-0480-6

3. Katz ES, D'Ambrosio CM. Pathophysiology of pediatric obstructive sleep apnea. Proc Am Thorac Soc . 2008;5(2):253-262. doi:10.1513/pats.200707-111MG

4. Chang SJ, Chae KY. Obstructive sleep apnea syndrome in children: Epidemiology, pathophysiology, diagnosis and sequelae. Korean J Pediatr . 2010;53(10):863-871. doi:10.3345/kjp.2010.53.10.863

5. Frye SS, Fernandez-Mendoza J, Calhoun SL, et al. Neurocognitive and behavioral functioning in adolescents with sleep-disordered breathing: A population-based, dual-energy X-ray absorptiometry study. Int $J$ Obes (Lond) . 2018;42(1):95-101. doi:10.1038/ijo.2017.229

6. Redline S, Tishler PV, Schluchter M, Aylor J, Clark K, Graham G. Risk factors for sleep-disordered breathing in children. Am J Respir Crit Care Med . 1999;159(5):1527-1532. doi:10.1164/ajrccm.159.5.9809079

7. Ross KR, Storfer-Isser A, Hart MA, et al. Sleep disordered breathing is associated with asthma severity in children. J Pediatr . 2012;160(5):736-742. doi:10.1016/j.jpeds.2011.10.008 
8. Sánchez T, Castro-Rodríguez JA, Brockmann PE. Sleep-disordered breathing in children with asthma: a systematic review on the impact of treatment. J Asthma Allergy . 2016;9:83-91. doi:10.2147/JAA.S85624

9. Emancipator JL, Storfer-Isser A, Taylor HG, et al. Variation of cognition and achievement with sleepdisordered breathing in full-term and preterm children. Arch Pediatr Adolesc Med . 2006;160(2):203. doi:10.1001/archpedi.160.2.203

10. Sadras I, Reiter J, Fuchs N, Erlichman I, Gozal D, Gileles-Hillel A. Prematurity as a risk factor of sleepdisordered breathing in children younger than two years: a retrospective case-control Study. J Clin Sleep Med . doi: $10.5664 / j c s m .8072$

11. Hunter SJ, Gozal D, Smith DL, Philby MF, Kaylegian J, Kheirandish-Gozal L. Effect of sleep-disordered breathing severity on cognitive performance measures in a large community cohort of young school-aged children. Am J Respir Crit Care Med . 2016;194(6):739-747. doi:10.1164/rccm.201510-2099OC

12. Marcus CL, Moore RH, Rosen CL, et al. A randomized trial of adenotonsillectomy for childhood sleep apnea. N. Engl. J. Med . 2013;368(25):2366-2376. doi:10.1056/NEJMoa1215881

13. Spruyt K, Gozal D. A mediation model linking body weight, cognition, and sleep-disordered breathing. Am J Respir Crit Care Med . 2012;185(2):199-205. doi:10.1164/rccm.201104-0721OC

14. Bourke R, Anderson V, Yang JSC, et al. Cognitive and academic functions are impaired in children with all severities of sleep-disordered breathing. Sleep Medicine . 2011;12(5):489-496. doi:10.1016/j.sleep.2010.11.010

15. Pietropaoli N, Supino MC, Vitelli O, et al. Cognitive function in preschool children with sleep-disordered breathing. Sleep Breath . 2015;19(4):1431-1437. doi:10.1007/s11325-015-1157-3

16. Owens J, Spirito A, Marcotte A, McGuinn M, Berkelhammer L. Neuropsychological and behavioral correlates of obstructive sleep apnea syndrome in children: a preliminary study. Sleep Breath . 2000;4(2):6777. doi:10.1007/BF03045026

17. O'Brien LM, Mervis CB, Holbrook CR, et al. Neurobehavioral correlates of sleep-disordered breathing in children. J Sleep Res . 2004;13(2):165-172. doi:https://doi.org/10.1111/j.1365-2869.2004.00395.x

18. Biggs SN, Walter LM, Jackman AR, et al. Long-term cognitive and behavioral outcomes following resolution of sleep disordered breathing in preschool children. PLOS ONE . 2015;10(9):e0139142. doi:10.1371/journal.pone.0139142

19. Gottlieb DJ, Chase C, Vezina RM, et al. Sleep-disordered breathing symptoms are associated with poorer cognitive function in 5-year-old children. J. Pediatr . 2004;145(4):458-464. doi:10.1016/j.jpeds.2004.05.039

20. Archbold KH, Giordani B, Ruzicka DL, Chervin RD. Cognitive executive dysfunction in children with mild sleep-disordered breathing. Biol Res Nurs . 2004;5(3):168-176. doi:10.1177/1099800403260261

21. Beebe DW, Ris MD, Kramer ME, Long E, Amin R. The association between sleep disordered breathing, academic grades, and cognitive and behavioral functioning among overweight subjects during middle to late childhood. Sleep . 2010;33(11):1447-1456. doi:10.1093/sleep/33.11.1447

22. Wechsler D. Wechsler Abbreviated Scale of Intelligence. New York: Psychological Corporation; 1999.

23. Wechsler D. Wechsler Intelligence Scale for Children-Third Edition Manual . San Antonio, TX: Psychological Corporation; 1991.

24. Wechsler D. WISC-IV Technical Manual . San Antonio, TX: Psychological Corporation; 2003.

25. Roid GH. Stanford-Binet Intelligence Scales, fifth edition . Rolling Meadows, IL: Riverside Publishing; 2003.

26. Merz WR. Kaufman Assessment Battery for Children . Kansas City, Mo: Test Corp America; 1984. 
27. Wechsler D. WPPSI-III technical and interpretive manual . San Antonio, TX: The Psychological Corporation; 2002.

28. Gozal D, Crabtree VM, Sans Capdevila O, Witcher LA, Kheirandish-Gozal L. C-reactive protein, obstructive sleep apnea, and cognitive dysfunction in school-aged children. Am J Respir Crit Care Med . 2007;176(2):188-193. doi:10.1164/rccm.200610-1519OC

29. Smithson L, Baird T, Tamana SK, et al. Shorter sleep duration is associated with reduced cognitive development at two years of age. Sleep Medicine . 2018;48:131-139. doi:10.1016/j.sleep.2018.04.005

30. Smith DL, Gozal D, Hunter SJ, Kheirandish-Gozal L. Frequency of snoring, rather than apnea-hypopnea index, predicts both cognitive and behavioral problems in young children. Sleep Medicine . 2017;34:170-178. doi:10.1016/j.sleep.2017.02.028

31. Williams KT. AGS Expressive Vocabulary Test manual . Circle Pines, MN: American Guidance Service; 1997.

32. Korkman M, Kirk U, Kemp S. NEPSY: A Developmental Neuropsychological Assessment: Manual . San Antonio, TX: Psychological Corporation; 1998.

33. Dunn LM, Dunn LM. Examiner's manual for the PPVT-III, Peabody Picture Vocabulary Test. 3rd ed . Circle Pines, MN: American Guidance Service; 1997.

34. Jackman AR, Biggs SN, Walter LM, et al. Sleep-disordered breathing in preschool children is associated with behavioral, but not cognitive, impairments. Sleep Medicine . 2012;13(6):621-631. doi:10.1016/j.sleep.2012.01.013

35. Taylor HG, Bowen SR, Beebe DW, et al. Cognitive effects of adenotonsillectomy for obstructive sleep apnea. Pediatrics . 2016;138(2). doi:10.1542/peds.2015-4458

36. Joyce A, Dimitriou D. Sleep-disordered breathing and cognitive functioning in preschool children with and without Down syndrome. J Intellect Disabil Res . 2017;61(8):778-791. doi:https://doi.org/10.1111/jir.12387

37. Cohen MJ. Children's Memory Scale Manual . San Antonio, TX: Psychological Corporation; 1997.

38. Delis DC, Kramer JH, Kaplan E, Ober BA. California Verbal Learning Test Manual . San Antonio, TX: Psychological Corporation; 1994.

39. Kheirandish-Gozal L, De Jong MR, Spruyt K, Chamuleau SAJ, Gozal D. Obstructive sleep apnoea is associated with impaired pictorial memory task acquisition and retention in children. Eur Respir $J$. 2010;36(1):164-169. doi:10.1183/09031936.00114209

40. Maski K, Steinhart E, Holbrook H, Katz ES, Kapur K, Stickgold R. Impaired memory consolidation in children with obstructive sleep disordered breathing. PLOS ONE . 2017;12(11):e0186915. doi:10.1371/journal.pone.0186915

41. Gregory AM, Caspi A, Moffitt TE, Poulton R. Sleep problems in childhood predict neuropsychological functioning in adolescence. Pediatrics . 2009;123(4):1171-1176. doi:10.1542/peds.2008-0825

42. Berry KE. The Beery-Buktenica Developmental Test of Visual Motor Integration: Administration, scoring and teaching manual . Parsippany, N J: Modern Curriculum Press; 1997

43.McCarthy D. Manual for the McCarthy Scales of Children's Abilities. San Antonio , TX: Psychological Corporation; 1972

44. Cooley EL, Morris RD. Attention in children: A neuropsychologically based model for assessment. Dev Neuropsychol . 1990;6(3):239-274. doi:10.1080/87565649009540465

45. Chervin RD. Sleep-disordered breathing, behavior, and cognition in children before and after adenotonsillectomy. Pediatrics . 2006;117(4):e769-e778. doi:10.1542/peds.2005-1837 
46. Christiansz JA, Lappin CR, Weichard AJ, et al. Slow wave activity and executive dysfunction in children with sleep disordered breathing. Sleep Breath . 2018;22(2):517-525. doi:10.1007/s11325-017-1570-x

47. Miyake A, Friedman NP, Emerson MJ, Witzki AH, Howerter A, Wager TD. The unity and diversity of executive functions and their contributions to complex "frontal lobe" tasks: A latent variable analysis. Cogn Psychol . 2000;41(1):49-100. doi:10.1006/cogp.1999.0734

48. Roye S, Castagna PJ, Calamia M, De Vito AN, Lee T-H, Greening SG. Relationships between multiple dimensions of executive functioning and resting-state networks in adults. Neuropsychologia . 2020;141:107418. doi:10.1016/j.neuropsychologia.2020.107418

49. Mietchen JJ, Bennett DP, Huff T, Hedges DW, Gale SD. Executive function in pediatric sleep-disordered breathing: a meta-analysis. J Int Neuropsychol Soc . 2016;22(8):839-850. doi:10.1017/S1355617716000643

50. Kaihua J, Yang Y, Fangqiao Z, Huijuan S, Chaoqun W, Xuan D. Event-related potentials and behavior performance scores in children with sleep-disordered breathing. Brain Dev . 2019;41(8):662-670. doi:10.1016/j.braindev.2019.04.008

51. Zahodne LB, Manly JJ, Smith J, Seeman T, Lachman ME. Socioeconomic, health, and psychosocial mediators of racial disparities in cognition in early, middle, and late adulthood. Psychol Aging.2017;32;118130. https://doi-org.proxy.uchicago.edu/10.1037/pag0000154

52. da Silva Gusmão Cardoso T, Pompéia S, Miranda MC. Cognitive and behavioral effects of obstructive sleep apnea syndrome in children: A systematic literature review. Sleep Medicine . 2018;46:46-55. doi:10.1016/j.sleep.2017.12.020

53. Smith DL, Gozal D, Hunter SJ, Kheirandish-Gozal L. Parent-reported behavioral and psychiatric problems mediate the relationship between sleep-disordered breathing and cognitive deficits in school-aged children. Front Neurol . 2017;8. doi:10.3389/fneur.2017.00410

54. Liu J, Liu X, Ji X, Wang Y, Zhou G, Chen X. Sleep disordered breathing symptoms and daytime sleepiness are associated with emotional problems and poor school performance in children. Psychiatry Res . 2016;242:218-225. doi:10.1016/j.psychres.2016.05.017

55. Stojek MMK, Montoya AK, Drescher CF, et al. Fitness, sleep-disordered breathing, symptoms of depression, and cognition in inactive overweight children: Mediation models. Public Health Rep . 2017;132:65S-73S. doi: $10.1177 / 0033354917731308$

56. Gozal D, Khalyfa A, Capdevila OS, Kheirandish-Gozal L, Khalyfa AA, Kim J. Cognitive function in prepubertal children with obstructive sleep apnea: a modifying role for NADPH oxidase p22 subunit gene polymorphisms? Antioxid Redox Signal . 2012;16(2):171-177. doi:10.1089/ars.2011.4189

57. Gozal D, Capdevila OS, Kheirandish-Gozal L, Crabtree VM. APOE epsilon 4 allele, cognitive dysfunction, and obstructive sleep apnea in children. Neurology . 2007;69(3):243-249. doi:10.1212/01.wnl.0000265818.88703.83 